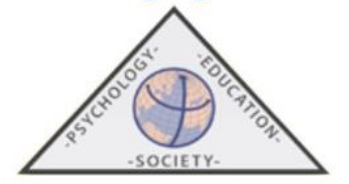

\title{
Construyendo ocios intergeneracionales, entre la vida y la escuela
}

\author{
Rita Gradaílle, José Antonio Caride y M. Belén Caballo \\ Universidad de Santiago de Compostela \\ Recibido el 18 de abril de 2021. Aceptado el 27 de julio de 2021
}

RESUMEN: Considerando que el ocio se ha convertido en un tema-problema de creciente interés en las Ciencias Sociales, asociamos sus desarrollos teórico-conceptuales, metodológicos y empíricos a las oportunidades que ofrecen los vínculos intergeneracionales para la construcción de una sociedad más inclusiva y equitativa, de y para todas las edades. Con la voluntad explícita de incorporar el ocio a los aprendizajes que procura la vida cotidiana, se pondrá énfasis en la importancia de las experiencias y vivencias que alientan los programas intergeneracionales en distintos contextos y realidades. Optando por un estudio de casos, se identifican, describen y analizan varias iniciativas protagonizadas por el alumnado y profesorado de distintos centros de Educación Primaria y Secundaria con personas mayores, muchas de ellas en situación de dependencia. Reconocidas como buenas prácticas premiadas por la Fundación DomusVi entre 2016 y 2020- muestran las potencialidades pedagógicas y sociales del ocio en las relaciones intergeneracionales, tanto en un plano individual como colectivo. Mejorar sus fundamentos teóricos, dotarlos de recursos institucionales y profesionales o dinamizar sus procesos son algunos de los desafíos que se plantean para garantizar su continuidad y sostenibilidad en el tiempo, mejorándonos como personas y sociedades.

Palabras clave: programas intergeneracionales; ocio; educación escolar; educación social; personas mayores.

\section{Building intergenerational leisure studies, between school and life}

ABSTRACT: Considering the fact that leisure time has become an increasingly interesting theme-problem in Social Sciences, we link its theoretical-conceptual, methodological and empirical developments to the opportunities offered by intergenerational bonds for the construction of a more inclusive and equitable society, from and for all ages. With the explicit will of incorporating leisure to the learnings sought after in daily life, the importance of the experiences that fuel intergenerational programs in different contexts and realities shall be emphasized. Opting for a study of cases, several initiatives protagonized by students and teachers from various Primary and Secondary schools with the elderly, many of them in a dependence situation, have been identified, described and analized. Recognized as good practices -having been awarded by the DomusVi Foundation between 2016 and 2020- they highlight the pedagogical and social potential of leisure time in intergenerational relationships, both from an individual and a collective perspective. Improving their theoretical basis, providing them with institutional and professional resources or boosting their processes are just some of the challenges raised to guarantee their continuity and sustainability over time, improving ourselves as people and as a society.

Keywords: intergenerational programmes; leisure; school education; social education; old people.

Correspondencia: Rita Gradaílle. Avda. Prof. Fráiz Andón, s/n, Facultad de CC. Educación, 15782 Universidad de Santiago de Compostela-Campus Vida. E-mail: rita.gradaille@usc.es 


\section{Introducción}

Las primeras aproximaciones sistemáticas al estudio del ocio adquirieron una especial relevancia a partir de los años centrales del siglo XX, vinculando sus desarrollos teórico-conceptuales a la investigación empírica, a la explicación y comprensión de sus realidades en relación con otros tiempos sociales (familiares y laborales), así como a la adopción de políticas públicas orientadas a promover prácticas recreativas saludables.

Será en las aportaciones realizadas por autores con perfiles y ámbitos científico- académicos diversos (Dumazedier, 1962; Grazia, 1962; Pieper, 1960; etc.) donde encontraron acomodo las principales indagaciones sobre la naturaleza y el alcance del ocio, inicialmente considerándolo como "un apéndice del trabajo" (Elias y Dunning, 1992, p. 117); más tarde, reconociendo su propia identidad, revisando los enfoques e hipótesis que se adoptaran epistemológica y metodológicamente. Con ellos renacían las imprecisas invocaciones al ocio como un derecho, alentado por las utopías renacentistas de Moro, Bacon o Campanella. Pero también los cuestionamientos que hicieran Lafargue (1880) o Veblen (1899), refiriéndose a las desigualdades sociales del capitalismo. De un modo u otro, se reflejarían en las primeras encuestas sobre los usos del tiempo, en los años veinte, para conocer y analizar los modos de vida en países tan dispares como Estados Unidos y la Unión Soviética, enfatizando la importancia de estudiar científicamente el tiempo desde una perspectiva social y cultural (Adam, 1990; Caride et al, 2020; Ramos, 1992; Sue, 1995; Tabboni, 2006).

Las transformaciones que acelera la globalización (Rosa, 2016; Wacjman, 2017), trazando nuevos horizontes para las interacciones y los vínculos sociales -a las que se añaden las adversidades pandémicas y socioambientales (covid-19, cambio climático, pérdida de biodiversidad, pobreza, etc.)modificará substancialmente los modos de apreciar y reivindicar los tiempos libres y, por extensión -sin equipararlos- los tiempos de ocio. También, inevitablemente, el tratamiento inter y transdisciplinar de sus temas-problema, dando respuesta a la complejidad que los caracteriza, con una doble misión (Barnett, 1988; Caride et al, 2020; Cuenca, 2018; Durán, 2007; Kleiber, 1999; Munné, 1980; Rosa, 2016; Safranski, 2017; Wajcman, 2017): de un lado, ampliar y diversificar los saberes que analizan, explican e interpretan las realidades del ocio en la sociedad-red en el desarrollo humano; de otro, mejorar los procesos orientados a la acción-intervención social mediante programas, proyectos, actividades, etc., desde su diseño hasta su evaluación en distintos contextos y realidades de la vida cotidiana.

Si hasta finales del siglo XX apenas ocupaban a la investigación social, los cambios acontecidos en las últimas décadas activaron el interés por las coordenadas espacio-temporales, con las potencialidades y riesgos que conlleva a nivel individual y colectivo. Abundan estudios, como los citados anteriormente, sobre la centralidad del tiempo y la sensibilidad hacia lo temporal en investigaciones sobre el trabajo, la familia, la equidad de género, la conciliación de la vida personal y profesional, las enseñanzas y los aprendizajes a lo largo de la vida, los medios de comunicación, etc. Desbordando la clásica distinción otium-negotium (Segura y Cuenca, 2007), el ocio alberga negocios hasta hace pocos años impensables.

Sus inquietudes figuran en las agendas de la investigación científica sobre el ocio, insistiendo en la necesidad de consensuar desde su concepto -por muchos todavía denostado- hasta la realización de estudios transversales, comparados y/o longitudinales, adoptando estrategias que permitan compensar desajustes, optimizando las posibilidades del ocio en los nuevos marcos de consumo y desarrollo tecnológico (Monteagudo, 2007). Las reflexiones sobre el ocio, dotando a sus discursos y prácticas de significados emergentes, además de afirmar su caracterización como un tiempo de libertades (Leif, 1992), obliga a una profunda relectura de sus ámbitos, así como de los impactos -beneficiosos y nocivosque genera en la economía, la educación, la vida familiar, la política, etc. 
No pueden ignorarse los usos y abusos que inducen los consumos masificados propiciados por un mercado global abierto las 24 horas, o la vulnerabilidad y fragilidad que perpetúan las desigualdades, la exclusión social, la dependencia y alienación de millones de personas, impidiendo o limitando su emancipación y/o autorrealización. No solo carecen de unos mínimos de bienestar físico, intelectual y emocional, sino también de la posibilidad de un pleno ejercicio de sus derechos cívicos (Caride, 2018), incluyendo "la felicidad de no hacer nada" (Schnabel, 2011). Y, por tanto, de un ocio al que se reconoce como un área específica de la experiencia humana con beneficios, como la libertad de elección, creatividad, satisfacción, disfrute y una mayor felicidad (WLRA, 1994).

\section{El Ocio y Las Edades: Nuevas Oportunidades Para La Convivencia Intergeneracional}

Siendo un componente substancial en los procesos de socialización, coincidimos con Negre (1993) en que la intensidad y diversidad del ocio tienen un componente generacional, convergente con nuevas formas de definir las conductas culturales personales que se orientan hacia objetivos singulares de cada sociedad (Touraine, 1971). La convivencia intergeneracional es una de ellas, cuando todo indica que en 2050 una de cada cinco personas tendrá 60 o más años.

El siglo XXI, demográficamente abocado a la superpoblación y a una esperanza de vida jamás alcanzada, está siendo el que más opciones ha deparado para el encuentro entre personas con distintas edades; y de aprovechar al máximo sus capacidades, según consta en el artículo 16 de la Declaración Política y Plan de Acción Internacional sobre Envejecimiento, para "fortalecer la solidaridad entre las generaciones y las asociaciones intergeneracionales, teniendo presentes las necesidades particulares de los más mayores y los más jóvenes y de alentar las relaciones solidarias entre generaciones" (Naciones Unidas, 2002, p. 4). En este contexto inscribe la educación intergeneracional sus prácticas, compartiendo los principios de una visión humanista, abierta a un aprendizaje permanente que asegure un porvenir sostenible y una existencia digna (UNESCO, 2015).

Conceptualmente, los programas intergeneracionales favorecen experiencias que promueven relaciones, intercambios, convivencias, conocimientos, etc. beneficiosos para quienes participan en su desarrollo, con la voluntad -más o menos explícita- de construir una sociedad para todas las edades, en la que no es suficiente con estar juntos ni la simple interacción: se requiere "hacer y hacerse juntos" (Newman y Sánchez, 2007, p. 42); de ahí la necesidad de generar espacios que faciliten el diálogo, la cooperación y la convivencia entre diferentes grupos de edad (Bagnasco et al, 2020; Canedo et al, 2017; Cohen-Mansfield y Jensen, 2017; Giraudeau y Bailly, 2019; Gualano et al, 2018; Kaplan, 2001; Peters et al, 2021; Zhong et al, 2020).

El diseño y la puesta en práctica de estas iniciativas enfatiza lo intergeneracional como un medio y un objetivo en sí mismo, para que las personas aprendan unas de otras, y promuevan valores como la participación y la solidaridad (Fundación FEDE, 2015). El protagonismo ciudadano, los procesos relacionales, la cohesión e inclusión social, así como el aprendizaje a lo largo de la vida son algunas de las motivaciones a las que se remiten, advirtiendo sobre la complejidad inherente a sus formas de conocer, decidir y actuar. Así, entre sus objetivos se contempla ampliar y diversificar las oportunidades en las relaciones de reciprocidad, rompiendo estereotipos, aprendiendo a respetar y admirar a una generación mayor; posibilitar la colaboración y el apoyo de la comunidad; fomentar la transmisión de tradiciones y valores a los más jóvenes, contribuyendo a la identidad en la diversidad; así como prevenir el aislamiento o la soledad de las personas mayores, estimulando cognitiva, emocional y socialmente su autoestima y la pertenencia a las redes que teje la sociedad.

Son muchas las iniciativas de ocio intergeneracional, protagonizadas por los abuelos/abuelas y nietos/nietas (Sanz et al, 2020), entre las que destacan los programas educativos en los que conviven niños y mayores; un tiempo que favorece la inclusión y la corresponsabilidad desde las primeras etapas 
de la vida, contribuyendo al envejecimiento satisfactorio de quienes tienen más edad (Cuenca, 2018; Monteagudo, 2020). Las responsabilidades familiares y de las escuelas en la educación del ocio no se cuestionan. Muy al contrario, crecen las evidencias que destacan el papel de las familias, del profesorado y del alumnado en la generación de beneficios -educativos, psicológicos y relacionales- vinculados con el bienestar de las personas y de las comunidades, desde la infancia hasta la vejez.

\section{El presente estudio}

La Fundación DomusVi se presenta como un espacio que impulsa, promueve y comparte el conocimiento y la formación entre todos sus profesionales y todas las personas del sector social y sanitario. Convoca desde 2016 distintos premios con el objetivo de reconocer el compromiso social de personas e instituciones para mejorar la calidad de vida de los mayores y en situación de dependencia, y lograr una sociedad más solidaria. Con varias modalidades y ámbitos de la acción social, la formación y la investigación, premian: escuelas de Educación Primaria y Secundaria; centros de Bachillerato y Formación Profesional; trabajos o proyectos de investigación sobre las personas en situación de dependencia (colectivo de personas mayores, salud mental y discapacidad); personas mayores activas y solidarias; profesionales del sector (mejor práctica en el cuidado de personas); medios de comunicación (rigor y sensibilidad hacia las personas mayores o en situación de dependencia); y la innovación en el sector socio-sanitario.

Sin obviar los vínculos institucionales que identifican la Fundación con la iniciativa privada, se trata de una línea de actuación pionera en nuestro país, consolidada en el tiempo (se ha convocado su sexta edición en 2021), contando con la participación de cientos de personas de todas las edades. Sin duda, merecedora de reconocimientos en todo lo que supone de aliento y visibilidad desde una perspectiva intergeneracional, inclusiva y dialogada. De ahí el interés en mostrar, con un propósito pedagógico y social, lo que estos premios representan en clave de enseñanzas y aprendizajes para difundir y motivar a otras entidades, instituciones, organizaciones, etc. con el fin de desarrollar proyectos intergeneracionales.

Partiendo de esta circunstancia, formulamos nuestro objetivo principal: identificar, describir y analizar experiencias educativas reconocidas como buenas prácticas en la promoción y el desarrollo de la convivencia intergeneracional. Se trata de poner en valor sus contribuciones a la formación en valores cívicos, respeto mutuo, saberes y aprendizajes compartidos, sensibilidad social y participación de la comunidad, etc., en distintas realidades, protagonizadas por niños/niñas, adolescentes y mayores, contribuyendo al logro de una sociedad para todas las personas y en todas las edades.

Este objetivo es convergente con los que figuran en programas intergeneracionales de todo el mundo y en las investigaciones que los han analizado en distintos países (Bagnasco et al, 2020; BCCPA, 2009; DFTA, 2010; Fundación FEDE, 2015; Generations United, 2021; Giraudeau y Bailly, 2019; Gutiérrez y Hernández, 2013; ICF-GHK, 2013; Newman y Sánchez, 2007; Orte y Vives, 2016; VillasBoas et al, 2017).

\section{Metodología}

Interpretando en clave de pasado estas iniciativas, aunque también con una visión prospectiva, metodológicamente optamos por realizar un estudio de casos múltiple, recopilando y analizando informaciones que tienen como nexo proyectos de acción-intervención de centros educativos con personas mayores de residencias y/o centros de día. Se trata de indagar, seleccionar y presentar brevemente experiencias que -desde un punto de vista educativo- destacan por su contribución a mejorar la calidad de vida de estas personas (muchas en situación de dependencia), favoreciendo relaciones empáticas con los niños/as y adolescentes. 
Como se sabe, el estudio de casos ayuda a conocer -con cierta profundidad- las realidades objeto de indagación, mediante análisis que aportan múltiples evidencias y perspectivas, aunque sus conclusiones no sean generalizables estadísticamente (Yin, 2009). Siendo estudios que no representan a un universo concreto, reflejan la singularidad y complejidad de cada caso, el alcance de sus iniciativas en contextos y/o circunstancias similares (Stake, 2005). El análisis documental de las experiencias premiadas por un jurado designado ad hoc, permitirá conocer los escenarios en los que han tenido lugar, con distintas miradas sobre la interacción y el aprendizaje intergeneracional.

\section{Participantes}

En el análisis que nos ocupa se han seleccionado catorce experiencias premiadas por la Fundación DomusVi (quien facilitó los expedientes de las experiencias premiadas, y autorizó su revisión para la elaboración de este artículo) en las cinco primeras ediciones (2016-2020) en las modalidades de "Escuelas de Educación Primaria y Secundaria: nuestros abuelos": 6 de Educación Primaria y 8 de Educación Secundaria de diferentes colegios de la geografía española, públicos $(n=4)$, privados $(n=2)$ y privados-concertados $(n=8)$. El galardón premia "la mejor iniciativa, proyecto, actividad, acto o campaña, de una escuela, colegio, curso, o clase del ciclo de Educación Primaria y Secundaria, que promueva entre los niños/as y adolescentes de Primaria y Secundaria, la educación en los valores de aprecio, respeto, cariño, admiración, generosidad, compromiso, agradecimiento hacia nuestros mayores, o llevando a cabo una iniciativa de interacción con personas mayores" (Fundación Domus Vi, 2021, s/p.).

En Educación Primaria son proyectos orientados a fomentar el diálogo intergeneracional, la convivencia y el aprendizaje de los niños y las personas mayores, promoviendo relaciones de empatía y respeto, creatividad, cooperación e innovación. En general, implican la participación del alumnado de los últimos cursos de esta etapa educativa y de mayores residentes, mediada por el profesorado y personal socio-sanitario de estos centros; en algunos casos, trascienden a los colectivos participantes, implicando a familiares y otras entidades del entorno comunitario.

Muchas son experiencias vinculadas al currículum escolar, contemplando un aprendizaje holístico de los contenidos trabajados en las aulas ligados a las historias de vida de los mayores; se favorece -con una metodología innovadora y actividades vinculadas al ocio- la asimilación de contenidos académicos de un modo experiencial, educando en valores y desarrollando la empatía y el respeto hacia los demás. Aunque, suelen realizarse en un trimestre en sesiones semanales de hora y media, algunos proyectos abarcaron todo el curso académico, con frecuencia más allá del horario lectivo.

Además de contribuir a una educación integral, afianzando conocimientos ligados a las costumbres y las tradiciones, desarrollan competencias y habilidades personales centradas en el diálogo, la perseverancia, la paciencia, la atención y la escucha por parte del alumnado; todas esenciales para estimular el pensamiento crítico, la inteligencia emocional o la promoción de relaciones intergeneracionales solidarias y empáticas. De ahí que, más allá de sus objetivos académico-curriculares, sean experiencias que contribuyen a mejorar la dimensión cognitiva de las personas mayores, su confianza y autoestima, posibilitando que sus conocimientos, recuerdos o vivencias sean portadores de aprendizajes para los menores, sobre todo en un plano personal y social, emocional y axiológico.

Las ocho experiencias premiadas en Educación Secundaria comparten el interés por fomentar los vínculos sociales entre jóvenes y mayores de residencias o centros de día próximos a los centros educativos. Dos son de titularidad pública y seis privados o privados-concertados. Mayoritariamente se sitúan en las Comunidades Autónomas de Cataluña y Aragón -tres en cada CCAA- una en la ciudad de Zamora y otra en Alicante. El alumnado cursaba de $2^{\circ}$ a $4^{\circ}$ de la ESO y solo en una de las experiencias se incorporan estudiantes de Formación Profesional de Grado Medio. Destaca la participación comprometida del profesorado y de profesionales de entidades en las que residen los ancianos, también 
del alumnado y de las personas mayores, pero sin implicar a agentes externos ni una proyección comunitaria significativa.

La mayoría son experiencias puntuales que, en algunos casos, se extienden a todo el trimestre. Vinculadas a diferentes materias del currículum proponen un enfoque lúdico y con metodologías participativas -para el alumnado y los mayores- en contextos de ocio. Dos mantienen la colaboración con entidades de mayores a través del voluntariado, propiciando un ocio solidario. En las ocho iniciativas los resultados han superado las expectativas iniciales de todos los participantes y, aunque no se contemplaba como un objetivo prioritario, el aspecto afectivo-emocional, las competencias sociales y relacionales son logros destacados.

\section{Resultados}

Del conjunto de experiencias premiadas por la Fundación DomusVi se han seleccionado dos de Educación Primaria (EP) y otras dos de Educación Secundaria (ES) para analizar con mayor profundidad, al cumplir un mayor número de criterios que las identifican como buenas prácticas (Gradaílle y Caballo, 2016); en concreto: innovación, sostenibilidad, replicabilidad y transferibilidad, transversalidad, implicación de la ciudadanía y trabajo en red, identidad y cohesión social, y evaluación comunitaria. Además, se ha buscado la equiparación en cuanto a la titularidad de los centros (dos públicos y dos privados concertados), contemplando la perspectiva territorial: dos en Cataluña, una en Aragón y otra en Galicia (ver Tabla 1).

\section{Tabla 1}

Identificación y descripción de las experiencias analizadas

\begin{tabular}{|c|c|c|c|}
\hline \multicolumn{4}{|c|}{ Educación Primaria } \\
\hline Experiencia & Agentes implicados & Temporalidad & Actividad \\
\hline \multirow[b]{2}{*}{ Ponte sus zapatos } & Casa Do Neno (alumnado) & & \multirow{4}{*}{$\begin{array}{l}\text { Proyectos, aprendizaje } \\
\text { cooperativo y rutinas de } \\
\text { pensamiento. }\end{array}$} \\
\hline & AMPAS & & \\
\hline $\begin{array}{l}\text { Santiago de } \\
\text { Compostela } \\
\text { (A Coruña) }\end{array}$ & $\begin{array}{l}\text { SarQuavitae } \\
\text { (mayores) }\end{array}$ & Seis semanas & \\
\hline 2017 & $\begin{array}{l}\text { Conservatorio Histórico } \\
\text { de Santiago }\end{array}$ & & \\
\hline Mayores y & Escola Carrilet & Curso 2018-2019 & \\
\hline pequeños, pequeños & (profesorado, alumnado de & & Participación en la $X^{a}$ \\
\hline y mayores & $4^{\circ}, 5^{\circ}$ y $6^{\circ}$, familias $)$ & $\begin{array}{l}\text { Un mes para el diseño } \\
\text { de la propuesta en una }\end{array}$ & $\begin{array}{l}\text { Edición del concurso } \\
\text { municipal Flors i Violes }\end{array}$ \\
\hline Palafrugell (Girona) & Fundación Palafrugell & comisón mixta & \\
\hline 2019 & $\begin{array}{l}\text { Gente Mayor } \\
\text { (profesionales, mayores) }\end{array}$ & Un mes de talleres & Talleres \\
\hline \multicolumn{4}{|c|}{ Educación Secundaria } \\
\hline Experiencia & Agentes implicados & Temporalidad & Actividad \\
\hline $\begin{array}{l}\text { Viu intensament la } \\
\text { música. Teràpia per } \\
\text { a jovens i grans! }\end{array}$ & $\begin{array}{l}\text { Escola Anna Mogas } \\
\text { (alumnado } 3^{\circ} \mathrm{ESO}, \\
\text { profesorado) }\end{array}$ & Desde 2009-2010 & \multirow{2}{*}{$\begin{array}{l}\text { Talleres vinculados a la } \\
\text { música }\end{array}$} \\
\hline $\begin{array}{l}\text { Granollers } \\
\text { (Barcelona) }\end{array}$ & $\begin{array}{l}\text { Residencias Mútuam de } \\
\text { les Franqueses y Can }\end{array}$ & Sesiones de 2 horas & \\
\hline
\end{tabular}




\begin{tabular}{|c|c|c|c|}
\hline 2016 & $\begin{array}{l}\text { Monich (mayores, } \\
\text { profesionales) }\end{array}$ & & \\
\hline & \multirow{5}{*}{$\begin{array}{l}\text { IES Reyes Católicos } \\
\text { (alumnado } 4^{\circ} \text { ESO, FP } \\
\text { grado medio; profesorado) } \\
\text { Residencia Municipal } \\
\text { Fundación Elvira Otal } \\
\text { (mayores, profesionales) }\end{array}$} & \multirow{5}{*}{$\begin{array}{l}\text { Curso 2018-2019 } \\
\text { Periodicidad semanal } \\
\text { durante } 3 \text { meses } \\
\text { ESO } 1 \text { hora semanal } \\
\text { FP, } 2 \text { horas semanales }\end{array}$} & $\begin{array}{l}\text { Encuentro inicial en el } \\
\text { instituto. }\end{array}$ \\
\hline $\begin{array}{l}\text { Nuestro jardín } \\
\text { terapéutico }\end{array}$ & & & ESO: diseño de proyecto \\
\hline Ejea de los & & & \\
\hline $\begin{array}{l}\text { Caballeros } \\
\text { (Zaragoza) }\end{array}$ & & & con los mayores \\
\hline 2019 & & & $\begin{array}{l}\text { FP: talleres de plantación y } \\
\text { mejora del huerto y jardín } \\
\text { con los mayores }\end{array}$ \\
\hline
\end{tabular}

Partiendo de los datos básicos de la Tabla 1, se presentan a continuación los resultados derivados del análisis de cada una de las iniciativas seleccionadas.

a) Ponte sus zapatos: Experiencia intergeneracional e interdisciplinar promovida por el colegio privado-concertado 'Casa do Neno' de Santiago de Compostela. Participan el centro educativo, la asociación de madres y padres del colegio, las personas residentes de SarQuavitae así como el Conservatorio Histórico de Santiago. La colaboración y coordinación con otros agentes, así como la diversidad de instituciones que intervienen en su implementación constituyen elementos esenciales para promover escuelas inclusivas que favorecen el respeto y la convivencia social. Se identifica como una buena práctica al implicar y cohesionar a la ciudadanía en torno a una iniciativa común, fomentando el trabajo en red.

Con una metodología innovadora basada en proyectos, el alumnado aprende contenidos de diferentes áreas de conocimiento y temáticas, desarrollando saberes y habilidades cada vez más valorados, empoderándolo y dándole las herramientas necesarias para resolver los conflictos. Una enseñanza transversal que educa para la vida desde una perspectiva integral; de ahí que mediante estrategias didáctico-metodológicas aprenden -a través del ocio- a compartir experiencias y vivencias personas mayores que actúan como maestros brindando sus conocimientos a los más pequeños. Esta colaboración promueve la creatividad, potencia la responsabilidad, incentiva la independencia de los menores y es fácilmente transferible y replicable en otros contextos educativos.

Entre las actividades diseñadas se encuentran los huertos tutorizados por sus abuelos y personas de la residencia, trabajando contenidos vinculados a las ciencias naturales, así como la tradición, poniendo en valor la propia cultura. Sus contenidos están aderezados con talleres de música y baile tradicional, dando a conocer a la infancia canciones y los bailes que forman parte del repertorio popular que acompañaba las labores agrícolas desempeñadas por los mayores; estas actividades se proyectaron más allá del colegio, implicando a otras entidades locales, como el Conservatorio de Santiago. También se diseñaron talleres de cocina para valorar los productos del huerto comunitario, además de favorecer una alimentación más saludable entre los pequeños. Una iniciativa que, por su diseño y configuración, es sostenible y viable en el tiempo. Su continuidad no compromete ni los recursos humanos, materiales ni económicos de los agentes participantes.

En este programa, además de la comunidad educativa (docentes, alumnado y familias) participan los usuarios de la residencia, los abuelos del alumnado y los vecinos de más edad del barrio; siendo así, el proyecto trasciende el recinto escolar y se transforma en comunitario, con logros gratificantes para todos los implicados.

b) Mayores y pequeños, pequeños y mayores: Desarrollada en el colegio público Carrilet (Palafrugell-Girona) en colaboración con la Fundación Palafrugell Gente Mayor con el objetivo de 
fomentar los vínculos y el trabajo compartido entre pequeños y mayores, posibilitando una implicación activa en la vida social, educativa y cultural de la comunidad de referencia. Se trata de un proyecto colectivo integrado y participado por diferentes agentes y miembros de la comunidad; lo que le confiere un carácter transversal en su implementación.

Una de sus grandes potencialidades radica en la creación de una comisión mixta donde pequeños y mayores comparten, consensuan y toman decisiones relativas al diseño, programación e implementación del proyecto 'Flor i Violes': una intervención paisajística que va más allá de adornar espacios del pueblo en primavera, valorando el trabajo cooperativo e intergeneracional al crear circuitos de patios -públicos y privados- que se convierten en escenarios de creaciones y decoraciones artísticas, conciertos y espectáculos ofrecidos a toda la comunidad.

Mediante la fusión de arte y naturaleza se crean espacios para la convivencia y la cohesión social, activando el compromiso y la asunción de responsabilidades individuales y colectivas en un proyecto de acción comunitaria. Se contribuye a mejorar la calidad de los procesos educativos en la vida cotidiana, la participación cultural y la transformación de la comunidad. Además, siendo una intervención holística e integrada hace uso de conocimientos, saberes, competencias y habilidades desde una perspectiva integral, con el fin de implementar acciones globales y entrelazadas. El impacto de las últimas ediciones se asocia a procesos de desarrollo endógeno generadas en el territorio, sostenibles y participadas por la ciudadanía.

c) Viu intensament la música. Teràpia per a jovens i grans! es un proyecto integral de musicoterapia que implica de forma activa a personas mayores de dos residencias y alumnado que cursa la asignatura de Música $-3^{\circ}$ de la ESO- en el colegio privado concertada Anna Mogas. Se diseña y desarrolla como una iniciativa de Aprendizaje-Servicio desde el trabajo en red de estas tres entidades, localizadas en la misma zona de Granollers.

Teniendo como objetivo principal mejorar la calidad de vida de los mayores a través de la musicoterapia, se trabajan aspectos físicos, cognitivos, emocionales y sociales, desarrollando un mayor compromiso social de los jóvenes con su entorno e implicándolos como agentes activos de la acción educativa. Se considera una buena práctica porque genera un impacto tangible en la calidad de vida de las personas y de las comunidades que habitan. Entre otros, satisface criterios relacionados con la implicación ciudadana y su progresivo empoderamiento en la medida en que los jóvenes incrementan su autonomía y responsabilidad, fortalecen la dimensión ética de su formación, trabajando valores de cooperación y ayuda mutua. Además, contribuyen a crear identidad y cohesión social, impulsando la convivencia intergeneracional y la promoción de la lengua y cultura catalanas desde experiencias relacionadas con la música.

Cabe señalar que esta iniciativa, con las adaptaciones pertinentes, es transferible a otros contextos y resulta sostenible en el tiempo. Aunque la duración sea breve ( 5 sesiones de 2 horas cada una), tiene continuidad, pues se ha mantenido desde su puesta en marcha, en el curso 2009-2010. También resulta un exponente de su estabilidad el hecho de que se integre en el Proyecto Educativo de Centro y que forme parte del Plan estratégico de la escuela en la medida en que conecta con uno de los principios de su ideario, centrado en la proyección de la escuela en el entorno, en el compromiso social y la colaboración con otras organizaciones. Se considera una experiencia de ocio exotélico de perfil lúdico y cultural, porque crea un ambiente distendido en el que mayores y jóvenes comparten vivencias relacionadas con la música.

d) La cuarta de las experiencias seleccionadas se desarrolló en el IES Reyes Católicos (Ejea de los Caballeros, Zaragoza) en el curso 2018-2019. Con el proyecto Nuestro jardín terapéutico se implica al alumnado de $4^{\circ}$ de ESO y $1^{\circ}$ del Grado Medio de FP en Producción Agropecuaria; profesorado de varias materias (producción agropecuaria, dibujo, servicios a la comunidad y economía); mayores de la Residencia Fundación Elvira Otal, la dirección del centro, terapeutas ocupacionales y un 
fisioterapeuta. El trabajo colaborativo entre personas de grupos etarios diferentes y de profesionales de distintas disciplinas evidencian el carácter transversal e integrador de la experiencia, implicando a diferentes agentes para -conjuntamente- diseñar y construir un jardín terapéutico, una mesa de cultivo y plantar flores en el "Jardín de los sentidos" de la residencia, concretamente en su unidad de Alzhéimer.

Su carácter innovador permite identificarla como una buena práctica, creando un espacio de trabajo compartido entre alumnado de FP y ESO, así como de personas mayores y profesionales de ambas entidades, confiriéndole una entidad singular a esta iniciativa de aprendizaje-servicio.

Además, cumple el criterio de eficacia y eficiencia, al crear un jardín terapéutico que rentabiliza los recursos de las entidades a través de su colaboración solidaria. La sostenibilidad también es un elemento destacado, al haberse construido y desarrollado a lo largo de tres meses, en sesiones semanales de una hora (alumnado de ESO) y de dos horas en FP. En la memoria de la iniciativa se da cuenta de su continuidad e impacto, ya que -finalizado el proyecto- los mayores siguen cuidando el jardín y se han creado vínculos afectivos entre jóvenes y mayores; en algunos casos se mantienen con iniciativas de voluntariado. Se trata de una experiencia original que inspira "el concepto de una acción transferible, sustentable e innovadora, homologable a determinados territorios" (Gradaílle y Caballo, 2016, p. 75).

\section{Discusión}

En las buenas prácticas analizadas, que vinculan los tiempos libres, el ocio y/o los aprendizajes curriculares, se constata la importancia socioeducativa y la viabilidad de iniciativas generadoras de espacios para la convivencia entre niños, jóvenes y mayores; también la colaboración entre instituciones y entidades que comparten un mismo territorio. Aunque, en general, tienen un carácter puntual y esporádico, son iniciativas que impulsan otras de mayor complejidad y estabilidad en el tiempo, vinculándolas con el Proyecto Educativo de los centros que las promueven. Todo indica que su sostenibilidad y continuidad, dotándolas de un carácter más integral, permitiría profundizar en los aprendizajes compartidos, visibilizar sus potencialidades para la convivencia y dotarlas de nuevas perspectivas en acción-intervención comunitaria.

Con todo, cabe señalar, que la mayoría de las experiencias analizadas tienen un enfoque curricular, centrando los objetivos y el análisis de los logros en la visión del alumnado, siendo minoritarias las que asumen un enfoque más amplio, que integre la perspectiva de los mayores, más acorde con principios y criterios de actuación pedagógico-sociales, de animación sociocultural, aprendizaje-servicio, comunidades de aprendizaje, ciudades educadoras o educación del ocio en contextos comunitarios (Colom y Orte, 2001; Martínez de Miguel et al, 2017; Sáez, 2002).

En este sentido, y para futuros estudios, sería deseable no ceñirse sólo a una revisión documental, sino completarla con información obtenida mediante un trabajo de campo específico. Situar estas iniciativas intergeneracionales en un marco que contemple el ocio educativo y el escenario comunitario multiplicaría su potencialidad; situándolas en la perspectiva de una educación a lo largo de la vida para lograr sociedades más justas e inclusivas, en consonancia con los Objetivos de Desarrollo Sostenible (ODS).

Los programas intergeneracionales deben ser uno de los principales caminos para transitar hacia el intercambio de recursos, vivencias, enseñanzas y aprendizajes... entre las personas de más edad y las más jóvenes, considerando el impacto que tienen en los modos de percibirse, incrementar su autoestima, mejorar sus procesos de socialización, contribuir a su salud física y mental, etc. ya sea en contextos comunitarios, institucionales y/o familiares (Canedo, García y Pacheco, 2017; Cohen-Mansfield y Jensen, 2017; Giraudeau y Bailly, 2019; Gualano et al, 2018; Sánchez et al, 2018; Campillo, Sánchez y Díaz, 2020; Zhong et al, 2020). 
Las evidencias que aporta la literatura científica y el análisis de experiencias intergeneracionales como las presentadas, junto con los programas intergeneracionales que promueven un ocio activo, destacan los beneficios para todas las personas que participan en sus iniciativas, ya sea en los centros educativos o en los distintos ámbitos (residencias, centros de día, comunidades, etc.) en los que se desarrollan, aunque requieren ser planificados con sumo cuidado; procurando el máximo protagonismo a quienes intervienen -en particular a los niños/niñas, adolescentes y mayores- desde su diseño hasta su evaluación, con el fin de garantizar su sostenibilidad en el tiempo, tender puentes entre la escuela y la vida cotidiana, el pasado-presente y el futuro, en cada persona y en los diferentes entornos en los que proyecta su convivencia. El ocio, la educación escolar y social, los aprendizajes colaborativos y la voluntad de crear una sociedad de, para y con todas las personas nos desafía como nunca antes; de ahí el compromiso pedagógico y social de todos, y muy especialmente a quienes hemos asumido la responsabilidad de mejorarla a través de la educación y el quehacer cívico-social.

\section{Agradecimientos}

Proyecto I+D+i «Educación y conciliación para la equidad: análisis de su incidencia en los tiempos escolares y sociales de la infancia» (Ref.: RTI2018-094764-B-I00), financiado por el Ministerio de Ciencia, Innovación y Universidades y el Fondo Europeo de Desarrollo Regional.

\section{Referencias}

Adam, B. (1990). Time and social theory. Oxford University Press.

Bagnasco, A., Hayter, M., Rossi, S., Zanini, M.P., Pellegrini, R., Aleo, G., Catania, G. y Sasso, L. (2020). Experiences of participating in intergenerational interventions in older people's care settings: A systematic review and meta-synthesis of qualitative literature. Journal of Advanced Nursing, 76(1), 22-33. https://doi.org/10.1111/jan.14214

Barnett, L. A. (Ed.) (1988). Research about leisure: past, present and future. Sagamore Publishing.

BCCPA (2009). Creating caring communities: a guide to establishing intergenerational programs for schools, care facilities and community groups. GT Publishing Services.

Brasileiro, M., Moreira, M., Fabricio, F., Barros, K., Alves, M., Medeiros, R., Sá, R. y Silva, A (2019). Intergenerational Programs among Children, Young and Elderly People with Educational Emphasis: An Integrative Review. HSO Journal of Gerontology \& Geriatric Medicine, 5(25), 18. https://doi.org/10.24966/GGM-8662/100025

Campillo, M., Sánchez, M. y Díaz, P. (2020). Present and future of school intergenerational programmes: A study from Spain. Educational Research, 62(4), 375-389. https://doi.org/10.1080/00131881.2020.1819847

Canedo, A., García, J. N., y Pacheco, D. I. (2017). A Systematic Review of the Effectiveness of $\begin{array}{lllll}\text { Intergenerational Programs. Frontiers in Psychology, } & \text { 8, } & 1882 .\end{array}$ https://doi.org/10.3389/fpsyg.2017.01882

Caride, J. A. (2012). Lo que el tiempo esconde, o cuando lo social necesita de la Pedagogía. Pedagogía Social. Revista Interuniversitaria, 32, 17-29. https://doi.org/10.7179/PSRI_2018.32.02

Caride, J. A., Caballo, M. B. y Gradaílle, R. (Coords.). (2020). Tiempos, educación y ocio en una sociedad de redes. Octaedro

Cohen-Mansfield, J. y Jensen, B. (2017). Intergenerational Programs in Schools. Journal of Applied Gerontology: the Official Journal of the Southern Gerontological Society, 36(3), 254-276. https://doi.org/10.1177/0733464815570663

Colom, A. J. y Orte, C. (Coords.) (2001). Gerontología educativa y social: pedagogía social y personas mayores. Universitat Les Illes Balears. 
Cuenca, M. (2018). Ocio valioso para un envejecimiento activo y satisfactorio. CCS.

DFTA (2010). Good practices in Intergenerational Programming: models advancing Policy, Practice and Research. Department for the Aging.

Dumazedier, J. (1962). Vers une civilisation du loisir? Seuil.

Durán, M. A. (2007). El valor del tiempo: ¿cuántas horas te faltan al día? Espasa-Calpe.

Elias, N. y Dunning, E. (1992). Deporte y ocio en el proceso de civilización. FCE.

Fundación DomusVi (30 de marzo de 2021). Premios Fundación. https://www.domusvi.es/premiosfundacion/

Fundación FEDE (2015). Hacia una sociedad intergeneracional: ¿ cómo impulsar programas para todas las edades? Guía práctica. Diputación Foral de Bizkaia.

Generations United (2021). Making the case for intergenerational programs. GU-RRF.

Giraudeau, C. y Bailly, N. (2019). Intergenerational programs: What can school-age children and older people expect from them? A systematic review. European Journal of Ageing, 16(3), 363-376. https://doi.org/10.1007/s10433-018-00497-4

Gradaílle, R. y Caballo, M.B. (2016). Las buenas prácticas como recurso para la acción comunitaria: criterios de identificación y búsqueda. Contextos Educativos, 19, 75-88. https://doi.org/10.18172/con.2773

Grazia, S. de (1962). Of Time, Work and Leisure. The Twentieth Century Fund.

Gualano, M., Voglino, G., Bert, F., Thomas, R., Camussi, E., y Siliquini, R. (2018). The impact of intergenerational programs on children and older adults: a review. International Psychogeriatrics, 30(4), 451-468. https://doi.org/10.1017/S104161021700182X

Gutiérrez, M. y Hernández, D. (2013). Los beneficios de los programas intergeneracionales desde la perspectiva de los profesionales. Pedagogía Social. Revista interuniversitaria, 21, 213-233.

ICF-GHK (2013). Learning for active ageing and intergenerational learning. Final report. DTI-CETecnhopolis.

Kaplan, M. S. (2001). School-Based Intergenerational Programs. IE-UNESCO.

Kleiber, D. (1999). Leisure experience and human development: a dialectic interpretation. Basic Books. Lafargue, P. (1880). Le droit à la paresse: refutation du droit au travail de 1848. L'Égalité.

Leif, J. (1992). Tiempo libre y tiempo para uno mismo: un reto educativo y cultural. Narcea.

Martínez de Miguel, S., Moreno, P. y Escarbajal, A. (coords.) (2017). Envejecimiento activo, programas intergeneracionales y educación social. Dykinson.

Monteagudo, M. J. (Ed.). (2007). El Ocio en la investigación actual: una lectura desde ámbitos, disciplinas, grupos de población y contextos geográficos. Universidad de Deusto.

Monteagudo, M. J. (2020). Factores determinantes del ocio de las personas mayores y su contribución al envejecimiento satisfactorio. En J.A. Caride, M.B. Caballo y R. Gradaílle (Coords.), Tiempos, educación y ocio en una sociedad de redes (pp. 153-169). Octaedro.

Munné, F. (1980). Psicosociología del tiempo libre: un enfoque crítico. Trillas.

Naciones Unidas (2002). Declaración Política y Plan de Acción Internacional de Madrid sobre el Envejecimiento. ONU.

Negre, P. (1993). El ocio y las edades: estilo de vida y oferta lúdica. Hacer.

Newman, S. y Sánchez, M. (2007). Los programas intergeneracionales: concepto, historia y modelos. En M. Sánchez (Dir.), Programas intergeneracionales: hacia una sociedad para todas las edades (pp. 37-69). Fundación La Caixa.

Orte, C. y Vives, M. (Eds.). (2016). Compartir la infancia. Proyectos intergeneracionales en las escuelas. Octaedro.

Peters, R., Ee, N., Ward, S., Kenning, G., Radford, K., Goldwater, M., Dodge, H., Lewis, E., Xu, Y., Kudrna, G., Hamilton, M., Peters, J., Anstey, K. Tautenschlager, N., Fitzgerald, A. y Rockwood, 
K. (2021). Intergenerational Programmes bringing together community dwelling non-familial older adults and children: A Systematic Review. Archives of gerontology and geriatrics, 94, 104356. https://doi.org/10.1016/j.archger.2021.104356

Pieper, J. (1960). Leisure: The Basis of Culture. Pantheon.

Ramos, R. (Coord.) (1992). Tiempo y sociedad. CIS.

Rosa, H. (2016). Alienación y aceleración: hacia una teoría crítica de la temporalidad en la modernidad tardía. Katz.

Sáez, J. (Coord.). (2002). Pedagogía Social y programas intergeneracionales: educación de personas mayores. Aljibe.

Safranski, R. (2017). Tiempo: la dimensión temporal y el arte de vivir. Tusquets.

Sánchez, M., Sáez, J., Díaz, P. y Campillo, M. (2018). Intergenerational Education in Spanish Primary Schools: Making the Policy Case. Journal of Intergenerational Relationships, 16(1-2), 166-183. https://doi.org/10.1080/15350770.2018.1404859

Sanz, E., Valdemoros, M.A., Ponce de León, A., Alonso, R.A. y Sáenz de Jubera, M. (2020). Ocio y bienestar en clave intergeneracional. En J.A. Caride, M.B. Caballo y R. Gradaílle (Coords.), Tiempos, educación y ocio en una sociedad de redes (pp. 139-152). Octaedro.

Schnabel, U. (2011). Ocio: la felicidad de no hacer nada. Plataforma.

Skate, R. E. (2005). Investigación con estudio de casos. Morata.

Segura, S. y Cuenca, M. (2007). El ocio en la Grecia clásica. Universidad de Deusto.

Sue, R. (1995). Temps et ordre social: sociologie des temps sociaux. PUF.

Tabboni, S. (2006). Temps sociaux. Armand Colin.

Touraine, A. (1971). Trabajo, ocios y sociedad. En J. Dumazedier (Ed.), Ocio y sociedad de clases (pp. 93-120). Fontanella.

UNESCO (2015). Replantear la educación: ¿Hacia un bien común mundial?. UNESCO.

Veblen, Th. (1899). The Theory of the Leisure Class: An Economic Study of Institutions. MacMillan Company.

Villas-Boas, S., Oliveira, A., Ramos, N. y Montero, I. (2017). A educação intergeracional no quadro da educação ao longo da vida e do envelhecimento ativo. Revista de Estudios e Investigación en Psicología y Educación, 5, 188-193. https://doi.org/10.17979/reipe.2017.0.05.2584

Wacjman, J. (2017). Esclavos del tiempo: vidas aceleradas en la era del capitalismo digital. Paidós.

ELRA (1994). International Charter for Leisure. World Leisure \& Recreation, 36(2), 41-45. https://doi.org/10.1080/10261133.1994.9673916

Yin, R. (2009). Case study research: design and methods. Sage.

Zhong, S., Lee, Ch., Foster, M. y Bian, J. (2020). Intergenerational communities: A systematic literature review of intergenerational interactions and older adults' health-related outcomes. Social Science \& Medicine (1982), 264, 113374. https://doi.org/10.1016/j.socscimed.2020.113374 